EPJ Web of Conferences 107, 10004 (2016)

DOI: $10.1051 /$ epjconf/201610710004

(C) Owned by the authors, published by EDP Sciences, 2016

\title{
Translational invariant shell model for $\Lambda$ hypernuclei
}

\author{
R.V. Jolos ${ }^{1}$, L. Majling ${ }^{1,2, a}$, and O. Majlingova ${ }^{3}$ \\ ${ }^{1}$ Bogoliubov Laboratory of Theoretical Physics, JINR, Dubna, Russia \\ ${ }^{2}$ Nuclear Physics Institute, Řez near Prague, Czech Republic \\ ${ }^{3}$ Czech Technical University, Prague, Czech Republic
}

\begin{abstract}
We extend shell model for $\Lambda$ hypernuclei suggested by Gal and Millener by including $2 \hbar \omega$ excitations in the translation invariant version to estimate yields of different hyperfragments from primary $p$-shell hypernuclei. We are inspired by the first successful experiment done at MAMI which opens way to study baryon decay of hypernuclei. We use quantum numbers of group $\mathrm{SU}(4),[f]$, and $\mathrm{SU}(3),(\lambda \mu)$, to classify basis wave functions and calculate coefficients of fractional parentage.
\end{abstract}

\section{Introduction: Hypernuclei}

The $\Lambda$ hypernucleus ${ }_{\Lambda}^{A} \mathrm{Z}$ is a bound system of $Z$ protons, $A-Z-1$ neutrons and one $\Lambda$ hyperon. The lifetime of the hypernucleus is about $2 \cdot 10^{-10} \mathrm{~s}$. This is one of the best examples of a nucleus with a new flavor (strangeness).

Investigation of the production and properties of hypernuclei is of growing importance for contemporary nuclear and particle physics. The subject of hypernuclear physics involves various aspects of modern theoretical and experimental physics. The peculiar behavior of matter containing strange quark raised many interesting problems. The existence of hypernuclei gives a third dimension to the traditional world of nuclei [1]. Hypernuclei not only bring a strangeness to nuclear physics, they provide a convenient laboratory for obtaining information about the hyperon-nucleon (YN) interaction and explore the full $\mathrm{SU}(3)$ (flavor) symmetry breaking baryon-baryon interaction both strong and weak.

The empirical information on $\mathrm{YN}$ scattering consists almost exclusively on the spin averaged characteristics, the spin structure of the YN interaction is mainly unknown. The only way to obtain the necessary information is hypernuclear spectroscopy, since the results of hypernuclear structure calculations are sensitive to the spin dependence of the YN interaction.

There are several hypernuclear research centers and groups with complementary investigative programs: KEK,J-PARC,JLab, KAOS@MAMI, HypHI@GSI, STAR@RHIC, Alice@LHC; planned are BM@N in Dubna and PANDA@FAIR.

Developments and highlights are reported at triennial International Conferences on Hypernuclear and Strange Particle Physics [2].

\footnotetext{
a e-mail: majling@ujf.cas.cz
}

\section{Hypernuclear spectroscopy}

The spectroscopy of hypernuclei started to develop very quickly in 70's. It was M. I. Podgoretsky [3] who first pointed out that there is a magic kaon momentum $q_{K}$, at which the strangeness exchange reaction

$$
\left(K^{-}, \pi^{-}\right) \quad q_{K} \approx 530 \mathrm{MeV} / \mathrm{c}, \quad \theta_{\pi} \approx 0^{\circ},
$$

transforms a target neutron at rest into a stationary $\Lambda$ particle. In this reaction $\Lambda$ simply replaces a neutron in the nucleus, without otherwise changing its wave function. This fact inspired H. Lipkin to introduce the notion of "Strangeness Analogue State" (SAS) in $\Lambda$ hypernuclei [4]. The term "analogue state" is used to refer to the state obtained by substituting a $p$-shell $\Lambda$ for a $p$-shell neutron in the same space-spin state.

Much important $\Lambda$-hypernuclear experimental work based on the idea of Podgoretsky was done by Bogdan Povh (Heidelberg Saclay Collaboration at CERN) [5] and by R. Chrien at BNL [6].

Extensive studies of hypernucleus production have been made using the missing-mass spectroscopy in meson or electron induced reactions

$$
{ }^{A+1} \mathrm{Z}(a, b){ }^{A+1} \mathrm{Z}^{\prime},
$$

where $(a, b)=(K, \pi)$, or $(\pi, K)$, or $\left(e, e^{\prime} K^{+}\right)$[7].

The elementary processes for three typical reactions in terms of today's (quark) nomenclature are as follows:

A:

$$
\left(K^{-}, \pi^{-}\right)
$$

$$
\begin{array}{ccccccc}
K^{-} & + & n & \rightarrow & \Lambda & + & \pi^{-} \\
(s \bar{u}) & + & (u d d) & \rightarrow & (u d s) & + & (\bar{u} d)
\end{array}
$$

An $s$ in a $K^{-}$is exchanged with a $d$ in a $n$ 
B:

$$
\begin{aligned}
& \pi^{+}+n \stackrel{\left(\pi^{+}, K^{+}\right)}{\rightarrow} \Lambda+K^{+} \\
& (u \bar{d})+(u d d) \rightarrow(u d s)+(\bar{s} u)
\end{aligned}
$$

C:

$$
\begin{aligned}
& \left(e, e^{\prime} K^{+}\right) \\
& p \rightarrow \Lambda+K^{+}
\end{aligned}
$$

An $s \bar{s}$ pair is created associatively

The accepted interpretation is that hypernucleus is produced in a nucleon-hole $\Lambda$-particle excited state when a nucleon in the target nucleus in a state $j_{n}$ is converted to the $\Lambda$ hyperon in a state $\ell$ :

$$
\mid\left(j_{n}^{-1} \ell_{\Lambda}\right)^{\Delta J} \otimes \Psi^{A}>\quad \ell=0 s, 1 p, 2 d, 2 s, \cdots,
$$

where $\Psi^{A}$ stands for the wave function of the ground state of the core nucleus. For one particle states we use "harmonic oscillator" notation. The $\Delta J$ depends on reaction (and kinematics) used. This description obvious in closed-shell nuclei can be also used for all $p$-shell hypernuclei $[8,9]$. The broad peaks observed will generally have detailed structure.

The systematic study of excitation energy spectra were conducted for all $p$-shell hypernuclei, for some mediumheavy as well as for ${ }_{\Lambda}^{208} \mathrm{~Pb}$. The $\Lambda$-nucleus spectrum provides a "textbook example" of single particle structure in nuclear physics, see Fig. 1.

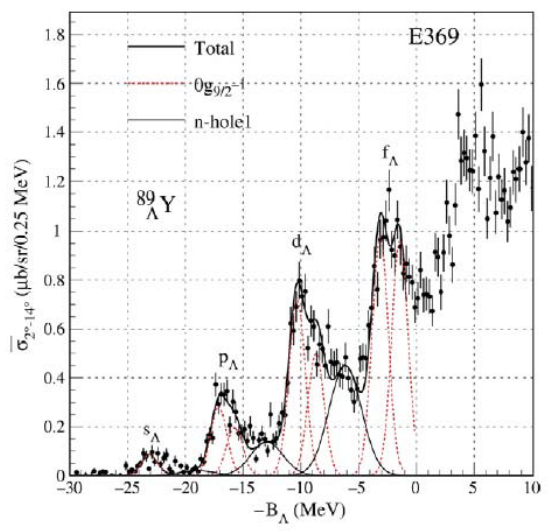

Figure 1. The single particle spectrum in ${ }_{\Lambda}^{89} \mathrm{Y}$, from [7].

Fifteen years ago, H. Tamura started a successful project of hypernuclear gamma spectroscopy with dedicated a germanium detector array [10]. A series of experiments on $p$-shell targets has been carried out using $\left(\pi^{+}, K^{+} \gamma\right)$ and $\left(K^{-}, \pi^{-} \gamma\right)$ reactions, [7]. A comprehensive shell model analysis of these $\gamma$-spectra was performed by Millener [11].

\section{Baryonic decay}

The full chain of events: formation of excited primary hypernucleus $\rightarrow$ baryon decay $\rightarrow$ weak decay of hyperfragment has been traced only in a single case [12], e.g.

$$
{ }_{\Lambda}^{12} \mathrm{C}^{*}(\mathrm{E} \sim 11 \mathrm{MeV}) \rightarrow p+{ }_{\Lambda}^{11} \mathrm{~B} .
$$

Recently, new projects were announced: two experiments at JLab [13] and at Mainz [14] intend to use high precision monochromatic $\pi^{-}$'s from the unique two-body weak decay to investigate light $\Lambda$ hypernuclei with variety of $(Z, A)$ combinations through identification of hyperfragments from strongly produced hypernuclear continuum in $\left(e, e^{\prime} K^{+}\right)$electro production. For example, with a ${ }^{7} \mathrm{Li}$ target the binding energies of ${ }_{\Lambda}^{3} \mathrm{H},{ }_{\Lambda}^{4} \mathrm{H},{ }_{\Lambda}^{5} \mathrm{H},{ }_{\Lambda}^{6} \mathrm{H},{ }_{\Lambda}^{6} \mathrm{He}$ and ${ }_{\Lambda}^{7} \mathrm{He}$ can be measured simultaneously.

The pioneering experiment was performed at MAMI, where a hypernuclear decay-pion study was realized using the A1 spectrometer facility with a ${ }^{9} \mathrm{Be}$ target and succeeded in the identification of the ${ }_{\Lambda}^{4} \mathrm{H}$ hyperfragment providing the feasibility of the method [15]:

$$
\begin{aligned}
& \text { production } \\
& { }^{9} \mathrm{Be}\left(e, e^{\prime} K^{+}\right){ }_{\Lambda}^{9} \mathrm{Li}^{*} \rightarrow \quad X+{ }_{\Lambda}^{4} \mathrm{H} \\
& \qquad{ }_{\Lambda}^{4} \mathrm{H} \rightarrow{ }^{4} \mathrm{He}+\pi^{-} \\
& \text {signature }
\end{aligned}
$$

The pion momentum distribution shows a monochromatic peak at $q_{\pi} \approx 133 \mathrm{MeV} / \mathrm{c}$, so, hyperfragment ${ }_{\Lambda}^{4} \mathrm{H}$ $\left(\mathrm{B}_{\Lambda}=2.12 \mathrm{MeV}\right)$ is identified uniquelly.

The experiment opens new chapter, namely systematical study of baryonic decays [16]. While missing mass experiments are limited to nuclei close to the initial target, the QF kaon production of an excited primary hypernucleus and its subsequent decay gives access to a variety of light and exotic hypernuclei, some of which cannot be produced or measured precisely by other means.

The experiment completes also hypernuclear spectroscopy in the $p$-shell. The comprehensive shell model approach was developed in [9]. At that time (80's) the attention was focused on $p_{n} \rightarrow p_{\Lambda}$ transitions seen in $\left(K^{-}, \pi^{-}\right)$ and $\left(\pi^{+}, K^{+}\right)$reactions. In line with nuclear structure terminology the states, in which $s_{\Lambda}\left(p_{\Lambda}\right)$ is coupled to a nuclear $p$-shell core, were called $0 \hbar \omega(1 \hbar \omega)$ excitations, respectively. There are also $1 \hbar \omega$ configurations of the form $s^{3} p^{k+1} s_{\Lambda}$ which can also be formed in reaction. These $s_{n} \rightarrow s_{\Lambda}$ transitions show up very clearly for the Li targets, see Fig. 2.

We have noted similarity of hypernuclear spectra with Giant Dipole Resonance [17], namely its "configurational splitting" [18], when nominally $1 \hbar \omega$ states are splitted into three groups, because single-particle energies differ significantly, as can be seen in Table 1 .

Table 1. Three groups of $1 \hbar \omega$ configurations

$$
\begin{array}{ll}
1 \hbar \omega_{\Lambda}\left(\varepsilon_{p_{\Lambda}}\right): & p^{-1} p_{\Lambda} \\
\begin{array}{l}
1 \hbar \omega_{N}\left(\varepsilon_{d}\right): \\
1 \hbar \omega_{N^{\prime}}\left(\varepsilon_{s^{-1}}\right):
\end{array} & \underbrace{-1 d s_{\Lambda}} s^{-1} p s_{\Lambda}
\end{array}
$$

The concept of configurational splitting comprises two effects [18, 20]:

(i) the energy gap between the groups of transitions corresponding to valence $(1 p \rightarrow 2 d, s)$ and core nucleon excitation $(0 s \rightarrow 1 p), \quad$ and 


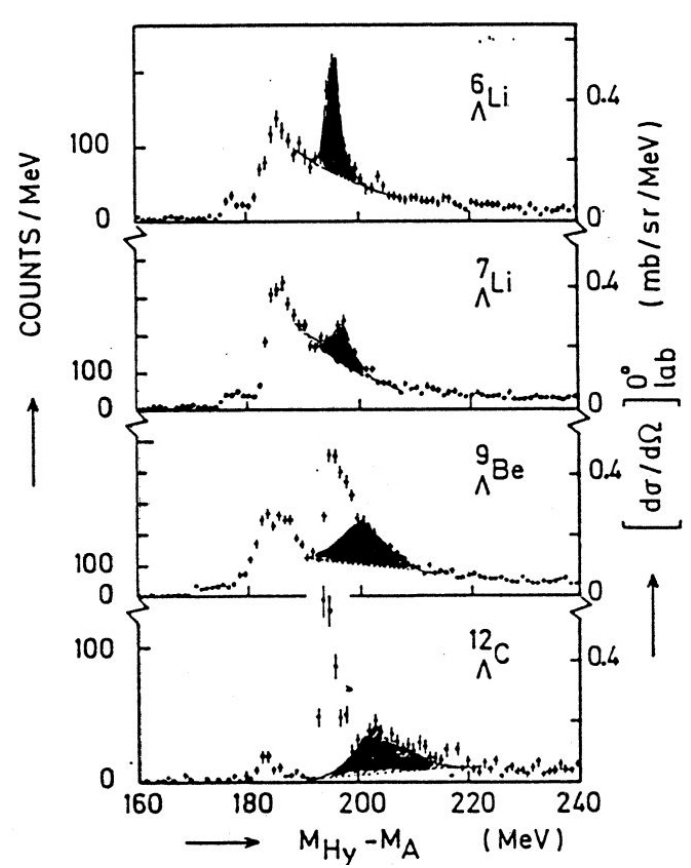

Figure 2. The $s^{-1} s_{\Lambda}$ states in $p$-shell nuclei, from [19].

(ii) the predominance of clustering phenomena in the lightest nuclei (related to Wigner supermultiplet symmetry).

The distinctive structure of the $s^{-1} s_{\Lambda}$ hypernuclear state influences strongly its decay properties. Calculations for $s^{-1} s_{\Lambda}$ states require careful treatment of center-ofmass, e.g. Translation Invariant Shell Model (TISM) [21] with its quantum numbers $[f]$ (Young tableaux) and $(\lambda, \mu)$ (Elliott's SU(3)). The emission of the $3 \mathrm{~N}$ cluster: ${ }^{3} \mathrm{He},{ }_{\Lambda}^{4} \mathrm{H}$ is the most probable.

For the first time, a benefit of the classification of highly-excited hypernuclear states in terms of $[f]$ and $(\lambda, \mu)$ was exemplified by decays of ${ }_{\Lambda}^{6} \mathrm{Li}$ [22], see Fig. 3. The same considerations may be applied [16] when we search for origin of the secondary $\gamma$ quanta $\left({ }_{\Lambda}^{7} \mathrm{Li}\right)$ observed in the experiment ${ }^{10} \mathrm{~B}\left(K^{-}, \pi^{-} \gamma\right)$ [7].

The consequences of the shell model structure of ${ }_{\Lambda}^{7} \mathrm{He}^{*}$ on its decay modes, i.e. the forbidden decay of the $s_{\Lambda} s^{-1}$ state to He isotopes is illustrated in Fig. 4.

We welcome the new project and already have discussed its potential in terms of SAS [23]. However, the excitation spectra of primary hypernucleus ${ }_{\Lambda}^{9} \mathrm{Li}$ in this production reaction is without any structure due to a small selectivity: spin-flip and large momentum, see Fig. 5.

There exist many open channels in ${ }_{\Lambda}^{9} \mathrm{Li}$ (see Table 2). Simulated decay-pion momentum spectrum (the relative yields) were calculated by a statistical decay model [24]. This model explicitly assumes the formation of an excited ${ }_{\Lambda}^{9} \mathrm{Li}^{*}$ which creates hypernuclei by fragmentation or deexcitation. It predicted three strong peaks, namely for ${ }_{\Lambda}^{7} \mathrm{He},{ }_{\Lambda}^{8} \mathrm{Li}$ and ${ }_{\Lambda}^{4} \mathrm{H}$ but the only last one was approved in experiment $[15,25]$.

In [27], we extended the TISM by including the next harmonic oscillator excitations $\left(\mathrm{N}_{\min }+2\right)$. Here, we discuss some steps in more detail.

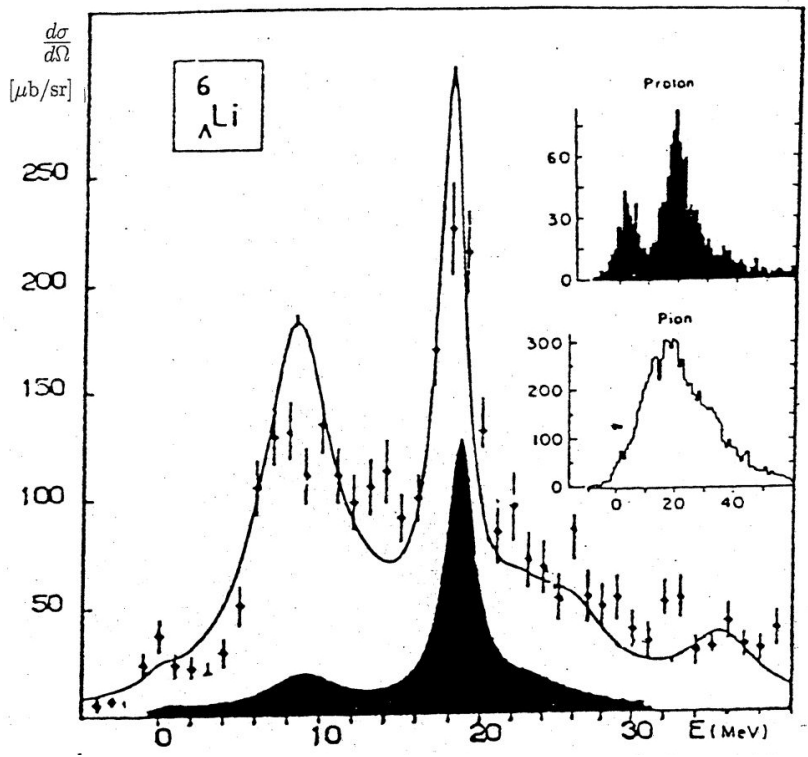

Figure 3. Spectrum of ${ }_{\Lambda}^{6} \mathrm{Li}$, from [22].

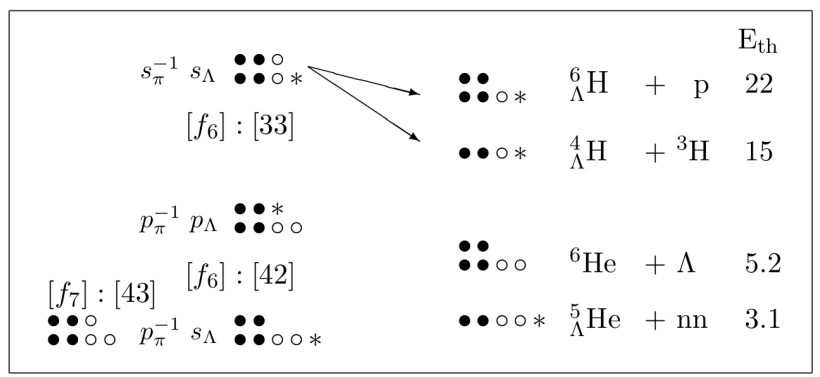

Figure 4. Schematic spectrum of ${ }_{\Lambda}^{7} \mathrm{He}: \bullet, \circ$ and $*$ marked neutron, proton and $\Lambda$, respectively; the $\left[f_{A}\right]$ stay for Young scheme.
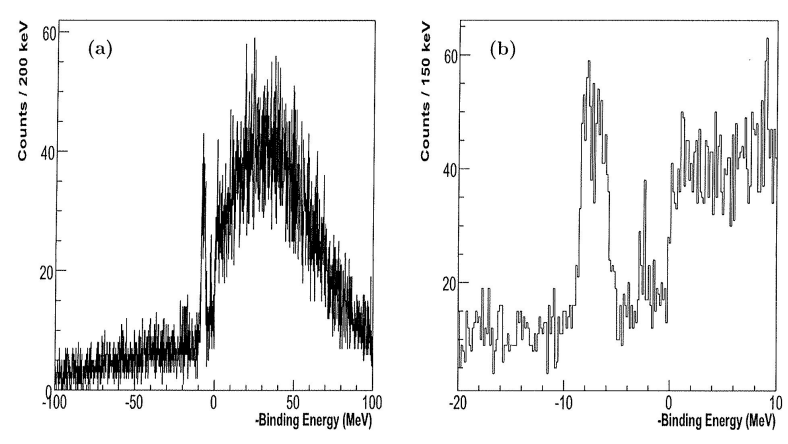

Figure 5. Excitation spectrum of ${ }_{\Lambda}^{9} \mathrm{Li}$ : the whole energy range (a) and the region of interest (b). From [26].

\section{TISM}

A small number of degrees of freedom involved to Nuclear Shell Model approved to be a very suitable instrument to analyze the experimental data in this field. First of all, shell model means an exact inclusion into consideration of the Pauli principle, since the basis of the shell model 
Table 2. Decays of ${ }_{\Lambda}^{9} \mathrm{Li}$

\begin{tabular}{|c|c|c|c|c|}
\hline & $\left({ }_{\Lambda}^{6} \mathrm{Li}\right)$ & ${ }_{\Lambda}^{7} \mathrm{Li}$ & ${ }_{\Lambda}^{8} \mathrm{Li}$ & ${ }_{\Lambda}^{9} \mathrm{Li}$ \\
& $3 n$ & $2 n$ & $n$ & $\Lambda$ \\
& 19.0 & 12.2 & 3.7 & 8.5 \\
\hline${ }_{\Lambda}^{4} \mathrm{He}$ & ${ }_{\Lambda}^{5} \mathrm{He}$ & ${ }_{\Lambda}^{6} \mathrm{He}$ & ${ }_{\Lambda}^{7} \mathrm{He}$ & ${ }_{\Lambda}^{8} \mathrm{He}$ \\
tnn & $t n$ & $t$ & $d$ & $p$ \\
31.5 & 9.9 & 9.7 & 13.0 & 13.8 \\
\hline${ }_{\Lambda}^{3} \mathrm{H}$ & ${ }_{\Lambda}^{4} \mathrm{H}$ & $\left({ }_{\Lambda}^{5} \mathrm{H}\right)$ & ${ }_{\Lambda}^{6} \mathrm{H}$ & $\left({ }_{\Lambda}^{7} \mathrm{H}\right)$ \\
${ }^{6} \mathrm{He}$ & ${ }^{5} \mathrm{He}$ & ${ }^{4} \mathrm{He}$ & ${ }^{3} \mathrm{He}$ & $2 p$ \\
18.2 & 11.8 & & 31.5 & \\
\hline
\end{tabular}

consists in the wave function antisymmetric with respect to the particle permutations. As a consequence a permutation group should be used to construct the basis wave functions. The shell model technique is very effective if the Harmonic Oscillator single particle wave functions are used for the construction of the total wave function. As a consequence the low-dimensional unitary groups $\mathrm{SU}(2)$, $\mathrm{SU}(3)$ and SU(4) come into the game. The tens of years of application of the shell model have shown that the simplest shell model configurations play a very important role as being in many cases the main components of the nuclear wave function [28]. The wave functions constructed using the quantum numbers of the permutation and unitary groups are very suitable for analysis of the possible cluster decomposition of the state of hyperfragment formed in the reaction and they give a possibility to calculate preformation factors for various decay channels.

To analyze the emission of hyperfragment ${ }_{\Lambda}^{4} \mathrm{H}$ from $s^{-1} s_{\Lambda}$ states of $p$-shell hypernuclei we start with the shell model in LS-coupling base [27]. We use harmonic oscillator wave functions characterized by Young diagram $\left[f_{A}\right]=\left[f_{1} f_{2} f_{3} \cdots\right]$ (it labels representation of permutation group $S_{A}$ with $\left.f_{1} \geq f_{2} \geq f_{3} \cdots\left(\sum f_{i}=A\right)\right)$. The orbital part we characterize by $(\lambda \mu)$ of SU(3) representation [29], the spin-isospin part by $\left[\tilde{f}_{A}\right]$ of $S U(4)$ representation. So, we can explore simple SU(3) and SU(4) recoupling technique, e.g. Clebsch Gordan coefficients (CGC). More details can be found in [30].

The main driving features of nuclear structure can be represented algebraically. We recognize the limitations of the symmetry approach. It can only account for gross properties, any detailed description required more involved numerical calculations. Symmetry techniques can be used as an appropriate starting point for detailed calculations.

\subsection{Center of mass}

For the states with several open shells, first of all we have to fix the wave function of center-of-mass motion, $\Psi_{N}\left(R_{A}\right)$. The unitary transformation from the standard shell model to TISM is performed using the Talmi-Moshinsky coefficients [31]. The anatomy of kinematic correlations induced by fixing center-of-mass motion is demonstrated clearly for hypernuclear wave function:

$$
\begin{array}{r}
\Psi_{N}\left(R_{A}\right) \psi_{n}\left(r_{\Lambda}\right)=\sum_{\mathcal{N}, v} T_{\mathcal{N}, v}^{N, n} \Phi_{\mathcal{N}}\left(R_{A \Lambda}\right) \varphi_{v}(\varrho) \\
\left(R_{A \Lambda} \equiv R_{A}+r_{\Lambda}, \quad \varrho \equiv R_{A}-r_{\Lambda} ; \quad N+n=\mathcal{N}+v\right) .
\end{array}
$$

For $1 \hbar \omega$ excitations we have very simple relations

\begin{tabular}{|l|cr|}
\hline$(10)$ & $\Phi_{0} \varphi_{1}$ & $\Phi_{1} \varphi_{0}$ \\
\hline$\Psi_{0} \psi_{1}=$ & $\alpha$ & $\beta$ \\
$\Psi_{1} \psi_{0}=$ & $\beta$ & $-\alpha$ \\
\hline
\end{tabular}

$$
\text { with } \alpha=\sqrt{\frac{A}{A+\mu}}, \quad \beta=\sqrt{\frac{\mu}{A+\mu}} \quad\left(\mu=\frac{m_{\Lambda}}{m}\right)
$$

For $2 \hbar \omega$ excitations, there are two possible $(\lambda \mu)$ : (20), (01) and transformation reads

\begin{tabular}{|l|ccc|}
\hline$(20)$ & $\Phi_{0} \varphi_{2}$ & $\Phi_{1} \varphi_{1}$ & $\Phi_{2} \varphi_{0}$ \\
\hline$\Psi_{0} \psi_{2}=$ & $\alpha^{2}$ & $\sqrt{2} \alpha \beta$ & $\beta^{2}$ \\
$\Psi_{1} \psi_{1}=$ & $\sqrt{2} \alpha \beta$ & $-\alpha^{2}+\beta^{2}$ & $-\sqrt{2} \alpha \beta$ \\
$\Psi_{2} \psi_{0}=$ & $\beta^{2}$ & $-\sqrt{2} \alpha \beta$ & $\alpha^{2}$ \\
\hline$(01)$ & \multicolumn{3}{|c}{$\Phi_{1} \varphi_{1}$} \\
$\Psi_{1} \psi_{1}=$ & \multicolumn{3}{|}{-1} \\
\hline
\end{tabular}

\subsection{Fractional parentage}

The key ingredient in the shell model calculations are coefficients of fractional parentage (cfp), coefficients of decomposition of antisymmetric wave function for $A$ particles

$$
\begin{aligned}
& \mid \ell^{A}:\left[f_{A}\right](\lambda \mu)_{A} L S T: J>= \\
& \mid 0 s^{k s}\left[f_{s}\right] ; 1 p^{k p}\left[f_{p}\right](\lambda \mu)_{p} ; k^{k l}\left[f_{l}\right](\lambda \mu)_{l}: \\
& \quad\left[f_{A}\right](\lambda \mu)_{A} L S T: J> \\
& =\sum_{n=0}^{A-4} \Phi_{N}^{(A)}\left[f_{A}\right](\lambda \mu)_{N} \cdot \Psi_{n}\left(R_{A}\right),
\end{aligned}
$$

where $\ell=0 s ; \quad 1 p ; \quad 2 d, 2 s ; \quad 3 f, 3 p ; \ldots$ with obvious constraints $k s+k p+k l=A,\left[f_{s}\right] \otimes\left[f_{p}\right] \otimes\left[f_{l}\right]=\left[f_{A}\right]$, and $(\lambda \mu)_{N} \otimes(n 0)=(\lambda \mu)_{A}$ on product of antisymmetric wave functions for $A_{1}$ and $A_{2}$ particles $\left(A_{1}+A_{2}=A\right)$ and wave function of their relative motion $\varphi_{v}(r)\left(r=R_{A_{1}}-R_{A_{2}}\right)$. For TISM (harmonic oscillator) wave function we have

$$
\begin{aligned}
\Phi_{N}^{(A)}[f](\lambda \mu)= & \sum \sqrt{\frac{n_{f 1} n_{f 2}}{n_{f}}} \cdot G_{L} \cdot G_{S T} \times \\
& \Phi_{N_{1}}^{\left(A_{1}\right)}\left[f_{1}\right](\lambda \mu)_{1} \cdot \Phi_{N_{2}}^{\left(A_{2}\right)}\left[f_{2}\right](\lambda \mu)_{2} \cdot \varphi_{v}(r),
\end{aligned}
$$

with constraints $N=N_{1}+N_{2}+v$. Here, spin-isospin cfp,

$$
\begin{gathered}
G_{S T} \equiv<\tau^{A_{1}}\left[\tilde{f}_{1}\right] S_{1} T_{1} ; \tau^{A_{2}}\left[\tilde{f}_{2}\right] S_{2} T_{2} \mid \tau^{A}[\tilde{f}] S T>= \\
\left(\begin{array}{cc|c}
{\left[\tilde{f}_{1}\right]} & {\left[\tilde{f}_{2}\right]} & {[\tilde{f}]} \\
S_{1} T_{1} & S_{2} T_{2} & S T
\end{array}\right),
\end{gathered}
$$

is CGC for group SU(4). Orbital cfp,

$$
G_{L} \equiv<l^{A_{1}}(\lambda \mu)_{1} L_{1} ; l^{A_{2}}(\lambda \mu)_{2} L_{2} \mid l^{A}(\lambda \mu) L>^{v},
$$


is Racah coefficient for group SU(3) which describes recoupling of three representations

$$
(\lambda \mu)_{1} \otimes(\lambda \mu)_{2} \otimes(\nu 0)=(\lambda \mu) .
$$

For nucleon clusters, $\mid s^{k}[k](00)>$, the $G_{L}$ is simple CGC:

$$
\left(\begin{array}{cc|c}
(\lambda \mu)_{1} & (\nu 0) & (\lambda \mu) \\
L_{1} & l & L
\end{array}\right)
$$

The weight factor $\frac{n_{f 1} n_{f 2}}{n_{f}}$ is the ratio of dimensions of representations of the symmetry groups $S_{A_{1}}, S_{A_{2}}, S_{A}$.

\section{Overlook and suggestions}

Dalitz and Levi Setti, [32], fifty years ago, discussed the possibility that $\Lambda$ hyperons could stabilize particleunstable nuclear cores of $\Lambda$ hypernuclei and thus allow studies of neutron rich baryonic systems beyond the nuclear drip line. The $\Lambda$ 's effectiveness to enhance binding is primarily connected with the Pauli principle from which it is exempt allowing it to occupy the lowest $0 s_{\Lambda}$ orbital. Several unbound-core $\Lambda$ hypernuclei have been identified in emulsion work, [33], see Table 3.

Table 3. Hypernuclei in emulsion

$\begin{array}{lcccccc}\text { core } & { }^{5} \mathrm{He} & { }^{5} \mathrm{Li} & { }^{6} \mathrm{Be} & { }^{7} \mathrm{He} & { }^{8} \mathrm{Be} & { }^{9} \mathrm{~B} \\ \text { decay } & n & p & 2 p & n & \alpha \alpha & p \\ \mathrm{E}_{\text {res }} & 0.8 & 1.7 & 1.4 & 0.4 & 0.1 & 0.2 \\ \mathrm{~B}_{\Lambda} & 4.2 & 4.5 & 5.2 & 7.2 & 6.7 & 8.9 \\ { }_{\Lambda}^{A} \mathrm{Z} & { }_{\Lambda}^{6} \mathrm{He} & { }_{\Lambda}^{6} \mathrm{Li} & { }_{\Lambda}^{7} \mathrm{Be} & { }_{\Lambda}^{8} \mathrm{He} & { }_{\Lambda}^{9} \mathrm{Be} & { }_{\Lambda}^{10} \mathrm{~B}\end{array}$

It was R. H. Dalitz [34] who highlighted peculiarity of heavy Hydrogen isotopes, see Table 4 (from [35]).

Table 4. Hydrogen isotopes

$\begin{array}{lcccc} & { }^{4} \mathrm{H} & { }^{5} \mathrm{H} & { }^{6} \mathrm{H} & { }^{7} \mathrm{H} \\ \text { decay } & n & 2 n & 3 n & 4 n \\ \text { E }_{\text {res }} & 3.0 & \mathbf{1 . 8} & 2.7 & \mathbf{0 . 8} \\ & { }_{\Lambda}^{5} \mathrm{H} & { }_{\Lambda}^{6} \mathrm{H} & { }_{\Lambda}^{7} \mathrm{H} & { }_{\Lambda}^{8} \mathrm{H}\end{array}$

Recent experiment by FINUDA Collaboration [36] stirred renewed interest in charting domains of particlestable neutron-rich $\Lambda$ hypernuclei particularly for unbound nuclear cores [37]. Gal and Millener have studied within a Shell Model approach several neutron-rich $\Lambda$ hypernuclei in the nuclear $p$ shell that could be formed in $\left(K^{-}, \pi^{+}\right)$ ([36]) or in $\left(\pi^{-}, K^{+}\right)$[38] reactions on stable nuclear targets.

The HYPERNIS (HYPERNeutron-rich hydrogen ISotopes) experiment @ NUCLOTRON (Dubna) also planed investigation of ${ }_{\Lambda}^{6} \mathrm{H}[39]$.

The ${ }_{\Lambda}^{6} \mathrm{H}$ is produced as one of hyperfragments in

$$
{ }^{7} \mathrm{Li}\left(e, e^{\prime} K^{+}\right){ }_{\Lambda}^{7} \mathrm{He}^{*} \rightarrow p+{ }_{\Lambda}^{6} \mathrm{H}
$$

In this experiment three hyper Hydrogen isotopes, ${ }_{\Lambda}^{3} \mathrm{H}$, ${ }_{\Lambda}^{4} \mathrm{H}$ and ${ }_{\Lambda}^{6} \mathrm{H}$ are identified simultaneously, ${ }_{\Lambda}^{4} \mathrm{H}$ serves as a benchmark. (A natural ${ }^{6,7} \mathrm{Li}$ target is available for installation [25]).

The values of $q_{\pi}$ are given in Table 5 .

Table 5. The values of $q_{\pi}$ for ${ }_{\Lambda}^{A} \mathrm{H}$

$$
\begin{array}{cccc} 
& { }_{\Lambda}^{3} \mathrm{H} & { }_{\Lambda}^{4} \mathrm{H} & { }_{\Lambda}^{6} \mathrm{H} \\
q_{\pi} & 114.3 & 132.9 & 134.8
\end{array}
$$

In MAMI set up it is possible to use various targets to studying ${ }_{\Lambda}^{6} \mathrm{H}$. In Table 6 , we collect threshold energies for possible targets (we take $\mathrm{B}_{\Lambda}\left({ }_{\Lambda}^{6} \mathrm{H}\right) \approx 4 \mathrm{MeV}$ ):

Table 6. Threshold energies

$\begin{array}{cccccc}\text { target } & \mathrm{HN} & & & & \mathrm{E}_{\text {th }} \\ { }^{7} \mathrm{Li} & { }_{\Lambda}^{7} \mathrm{He} \rightarrow{ }_{\Lambda}^{6} \mathrm{H}+{ }^{1} \mathrm{H} & \mathbf{2 4 . 3} \\ { }^{9} \mathrm{Be} & { }_{\Lambda}^{9} \mathrm{Li} \rightarrow{ }_{\Lambda}^{6} \mathrm{H}+{ }^{3} \mathrm{He} & \mathbf{3 1 . 5} \\ { }^{10} \mathrm{~B} & { }_{\Lambda}^{10} \mathrm{Be} \rightarrow{ }_{\Lambda}^{6} \mathrm{H}+{ }^{4} \mathrm{Li} & 52.0 \\ { }^{11} \mathrm{~B} & { }_{\Lambda}^{11} \mathrm{Be} \rightarrow{ }_{\Lambda}^{6} \mathrm{H}+{ }^{5} \mathrm{Li} & \mathbf{3 8 . 1}\end{array}$

The experiments at MAMI and at NUCLOTRON are complementary: both identify three hyper Hydrogen isotopes simultaneously. MAMI determines $\mathrm{B}_{\Lambda}$ while NUCLOTRON lifetime (and existence of isomer).

\section{Acknowledgements}

This work was supported from Votruba Blokhintsev program. Work of L.M. was supported by the Grant Agency of the Czech Republic under the grant No P2013/15/4301. Work of O.M. was supported by the grant LG 14001(INGO II). L.M. would like to express his sincere thanks to P. Bydžovsky and Liguang Tang inviting him to this challenging project, to S.N. Nakamura and J. Pochodzalla for their interest in even preliminary results. Special thanks are due to John Millener and Patrick Achenbach for their expertise.

\section{References}

[1] W. Greiner, Inter. J. Mod. Phys. E 5, 1 (1996)

[2] Hyp-XI (HYP2012), XI Intern. Conference on Hypernuclear and Strange Partical Physics, Barcelona, Spain, Nucl. Phys. A 914 (2013)

[3] M.I. Podgoretsky, Zh. Exp. Teor. Fiz. 44, 695 (1963); Sov. Phys. -JETP 17, 470 (1963)

[4] H.J. Lipkin, Phys. Rev. Lett. 14, 18 (1965)

[5] W.Bruckner et al., Phys. Lett. B 62, 481 (1976); B. Povh, Ann. Rev. Nucl. Part. Sci. 28, 1 (1978)

[6] R. Chrien et al., Phys. Lett. B 89, 31 (1979); R.E. Chrien, C.B. Dover, Ann. Rev. Nucl. Part. Sci. 39, 113 (1981)

[7] O. Hashimoto, H. Tamura, Progr. Part. Nucl. Phys. 57, 564 (2006) 
[8] A. Gal, J.M. Soper, R.H. Dalitz, Ann. Phys. (NY) 63, $53(1971)$

[9] E.H. Auerbach et al., Ann. Phys. (NY) 148, 381 (1983)

[10] H. Tamura, Nucl. Phys. A 691, 86c (2001)

[11] D.J. Millener, Topics in Strangeness Nuclear Physics, Lecture Notes in Phys. 724, eds. P. Bydžovský, A. Gal, and J. Mareš, (Springer, New York, 2007) p. 31.

[12] R.H. Dalitz, D.H. Davis, D.N. Tovee, Nucl. Phys. A 450, 311c (1986)

[13] L. Tang et al., Study of light hypernuclei by pionic decay, Proposal E-10-001, Jefferson Lab.

[14] J. Pochodzalla, Acta Phys. Polon. B 42, 833 (2011) (ARXIv:1101.2790, 2011)

[15] A. Esser et al., Phys. Rev. Lett. 114, 232501 (2015)

[16] L. Majling, Nucl. Phys. A 639, 125c (1998)

[17] J. Žofka et al., Sov. J. Part. Nucl. 22, 628 (1991); 28, 101 (1997)

[18] R. Eramzhyan et al., Phys. Rep. 136, 229 (1986)

[19] R. Bertini et al., Nucl. Phys. A 368, 365 (1981)

[20] H. Kissener, I. Rotter, N. Goncharova, Fortschritte der Physik 35, 277 (1987)

[21] I. Kurdyumov et al., Nucl. Phys. A 145, 593 (1970)

[22] L. Majling et al., Phys. Lett. B 92, 256 (1980)

[23] O. Majlingova, L. Majling, PoS (Baldin ISHEEP XXI) 101 (2012)
[24] A. Sanchez Lorente, A.S. Botvina, J. Pochodzalla, Phys. Lett. B 697, 222 (2011); A.S. Botvina et al., Nucl. Phys. A 881, 228 (2012)

[25] P. Achenbach et al., Nucl. Phys. A 881, 187 (2012)

[26] G.M. Urcioli et al. (JLab Hall A Collaboration), Phys. Rev. C 91, 034308 (2015)

[27] O. Majlingova et al., PoS (Baldin ISHEEP XXII) 129 (2015)

[28] C. W. Johnson, Phys. Rev. C 91, 034313 (2015)

[29] J.P. Elliott, Proc. Roy. Soc. A 245, 128 (1958); 245, 562 (1958)

[30] R.V. Jolos, L. Majling, and O. Majlingova, in preparation.

[31] I. Talmi, Helvetica Phys. Acta 25, 185 (1952); M. Moshinsky, Nucl. Phys. 13, 104 (1959); A. Gal, Ann. Phys. (NY) 49, 341 (1968)

[32] R.H. Dalitz, R. Levi Seti, Nuovo Cimento 30, 489 (1963)

[33] D.H. Davis, Nucl. Phys. A 754, 3c (2005)

[34] R.H. Dalitz, Nucl. Phys. A 754, 14c (2005)

[35] G. Audi et al., Nucl. Phys. A 729, 3 (2003)

[36] M. Agnello et al., Nucl. Phys. A 881, 269 (2012)

[37] A. Gal, D.J. Millener, Phys. Lett. B 725, 445 (2013)

[38] H. Sugimura et al., Phys. Lett. B 729, 39 (2014)

[39] A. V. Averyanov et al., Phys. At. Nucl. 71, 2101 (2008) 